\title{
La Administración Local en los Estados Unidos de América. Especial referencia a la ciudad
}

\author{
Zulima Sánchez Sánchez \\ Becaria de Investigación del Área de Derecho Administrativo de la Universidad de \\ Salamanca
}

Sumario: I. INTRODUCCIÓN. II. EL CONDADO. 1. Concepto. 2. Competencias de los Condados. 3. La organización del Condado. 4. Problemas de la figura del Condado: un futuro incierto. III. ENTES CREADOS CON UNA FINALIDAD CONCRETA: ESPECIAL REFERENCIA A LOS DISTRITOS ESPECIALES. 1. Los Distritos Especiales. 1.1. Concepto. 1.2. Organización de los Distritos Especiales. 2. Otros organismos con finalidades específicas. 2.1. Distritos Escolares Dependientes. 2.2. Áreas impositivas subordinadas a los Condados. 2.3. Las asociaciones de vecinos o ciudadanos. 2.4. Asociaciones de condominio, caseros y cooperativas. 2.5. Organizaciones para la planificación y coordinación de las áreas metropolitanas y otras áreas en el ámbito inferior al Estado. 3. Críticas. IV. MUNICIPALITIES. 1. Concepto. 2. Townships. 3. La ciudad. 3.1. El nacimiento del municipio. 3.2. La muerte del municipio. 3.3. Delegación de poderes. 3.3.1. La regla de Dillon. 3.3.2. El derecho de home-rule. 3.3.3. Competencias de la ciudad. 3.4. La organización de la ciudad: el Ayuntamiento.

\section{INTRODUCCIÓN}

En el Derecho americano no existe una clasificación ni definición de las distintas entidades locales que se haya aceptado por la mayoría de la doctrina. Incluso muchos autores se niegan a darla, ya que opinan que la diversidad de regulación que existe y las diferencias entre unos y otros tipos de entes nos harían dejar fuera del concepto a gran multitud de entes ${ }^{1}$.

De un modo descriptivo podemos decir que para el ciudadano americano la organización territorial del país se encuentra dividida en tres niveles: federal, estatal y local ${ }^{2}$.

A pesar de las diferencias entre Estados, debemos decir que los tipos de unidades que se encuentran en ellos siguen un esquema bastante simi-

1 Vid. G. W. Colman, State and Local Government and Public-private Partnerships, Connecticut, 1989 , pp. 19 a 59.

2 Vid. Roger H. Wells, American local government, McGraw-Hill, 1939, p. 1. 
lar y tienen unas características comunes que permiten agruparlas para que podamos tener una visión de conjunto.

La mayor parte de los estadounidenses viven bajo la jurisdicción de varios tipos de entes locales. Todos se encuentran bajo el nivel nacional (federal) y del Estado, pero, aparte de estos entes superiores, también existen otras unidades investidas de responsabilidades públicas: Condados, township, municipalities (city, town, village) y Distritos Especiales. A los tres primeros se les considera como entes de carácter general en cuanto a la variedad de funciones que prestan. Los últimos tienen una esfera de competencias muy reducidas y con fines muy concretos.

En el presente artículo pretendemos mostrar la división territorial en los Estados Unidos, haciendo una especial referencia a las unidades inferiores: Municipalities.

La razón de que prestemos más atención a estos entes es por la tradición y especial importancia que tienen en el entramado de la división territorial, a la vez que, al ser las más cercanas al ciudadano, nos pueden aclarar la situación en la que ellos mismos se encuentran y los derechos que la Nación americana les confiere en el desarrollo y organización del país.

Si bien los Estados que forman la Confederación gozan de la mayor parte de los poderes - salvo en algunas materias que la Constitución Americana reserva a la Federación-, no se puede olvidar la importancia que tuvieron a lo largo de la historia las ciudades y pueblos. Fueron éstos el germen de la Guerra de la Independencia contra la Corona inglesa y el origen de la nación americana, pues sus ayuntamientos acogieron a los revolucionarios que terminaron creando una nación independiente y dándose una Constitución propia. Pese a que la figura fuera perdiendo poderes para pasar éstos a los Estados (especialmente después de la promulgación de la Regla de Dillon en 1868), en la actualidad se están devolviendo funciones a las ciudades que no dudan en depositar parte de esos poderes en un nuevo tipo de entidad que crece desmesuradamente: los Distritos Especiales frutos, éstos, de la concepción práctica que inunda a la sociedad, a la política y a la vida en los Estados Unidos. Los Distritos Especiales y otras entidades menores se ocupan de dar soluciones precisas a necesidades concretas y son entes abiertos a la participación del ciudadano.

Pocos dudan ya de la crisis en que se encuentra la democracia representativa. En este sentido, en Estados Unidos se empieza a apostar por los Distritos Especiales como forma de devolver a los ciudadanos de esas 
ciudades de monstruosas dimensiones la conciencia de comunidad, tan necesaria para recuperar el abandonado sentimiento de nación que salió de los pequeños pueblos de Nueva Inglaterra ocupados por colonos a los que les unía su sentimiento de ciudadanía.

\section{EL CONDADO}

Como aclaramos en la introducción, el county es, junto con las municipalities o townships, una de las dos instituciones de carácter general dentro del conjunto de entidades locales norteamericanas.

\section{Concepto}

Si bien no existe una definición expresa podemos aventurarnos a decir que el Condado es una entidad local de carácter general cuya creación y competencias le son atribuidas por el Estado en el que extiende su territorio. Suele desempeñar funciones públicas de colaboración delegadas de forma expresa tanto por leyes estatales como por normas federales. El Condado carece de poderes naturales que le doten de autonomía para la gestión de sus propios intereses (inherent home rule). Éstos les pueden ser otorgados por el Estado de forma expresa mediante normas de rango constitucional o legislativo (constitutional o statutory home rule). En la práctica muy pocos son los Condados que cuentan con este poder.

La figura del Condado cuenta con una gran tradición histórica en la organización territorial de los Estados Unidos. La llegada de los primeros colonos se produjo en 1620 y ya en 1643 se creó el primer Condado en Massachusetts. Desde ese año hasta nuestros días los Condados han evolucionado de forma distinta en cada Estado, si bien no debemos olvidar que su mayor o menor importancia depende, fundamentalmente, de motivos históricos y del papel que desempeñaran incluso antes de que se constituyese la nación americana tal y como la conocemos en la actualidad.

Por eso no es de extrañar que esta forma de gobierno sea más importante en el sudoeste y los Estados del centro del litoral Atlántico donde históricamente tuvo una relevancia primordial. En cambio, en los Estados del centro oeste, en Nueva York, Nueva Jersey y Pennsylvania las funciones se dividen entre los Condados y Municipalities, siendo esta última la que cuenta con más poderes debido al papel esencial que jugó en la historia de la fundación de los Estados Unidos y en el logro de la independencia con respecto a la Corona británica que luego veremos. 
A excepción de Rhode Island, se puede decir que la entidad del Condado aparece en todos los Estados de la Federación, ya que su importancia es esencial en la prestación de servicios sobre todo en las zonas rurales ${ }^{3}$.

\section{Competencias de los Condados}

Siguiendo la definición que hemos dado:

"El Condado es una entidad local de carácter general cuya creación y competencias le son atribuidas por el Estado en el que extiende su territorio.»

Diremos que estas competencias son atribuidas bien por la Cámara Legislativa o por la Constitución de cada Estado ${ }^{4}$. Estas normas deben reconocer un mínimo de competencias: los condados deben recaudar impuestos (inherent taxing power), emitir bonos y encargarse de los gastos necesarios para llevar a cabo las funciones y tareas que le son propias ${ }^{5}$. Estas tareas incluyen dirección de hospitales, bibliotecas, parques, aeropuertos, carreteras secundarias y puentes.

\section{En la definición también dijimos que el Condado:}

«... Suele desempeñar funciones públicas de colaboración delegadas de forma expresa tanto por leyes estatales como por normas federales».

Por delegación de los Estados los Condados tienen funciones como la de administración judicial: se encargan de aplicar las normas promulgadas tanto por el Estado como por otros entes locales; de la organización electoral; recaudación de impuestos; y funciones administrativas y de control de instituciones de asistencia social e instituciones correccionales. Otra de sus funciones es la de actuar como registro en materia de gastos, escrituras, arrendamientos, hipotecas y otro tipo de estudios estadísticos importantes.

\footnotetext{
${ }^{3}$ Los Condados también prestan servicios en algunas áreas que no pertenecen a un tipo de entidad concreto. Es el caso del Condado de Los Ángeles en California que ocupa un extenso territorio adyacente a la ciudad de Los Ángeles, pero que nunca se ha unido a ésta. En otros casos los servicios llegan a prestarse en zonas que están también bajo el control de una ciudad como sucede en el Condado de Dade en Florida, donde el gobierno del Condado presta ciertos servicios para todo el distrito metropolitano de Miami.

${ }^{4}$ Las excepciones a este principio general de total dependencia son muy escasas. Las veremos más adelante pero tenemos que decir que tan sólo 7 Estados ban aceptado la forma de home rule para que los Condados determinen su propia estructura.
}

5 Vid. J. F. Dillon, Comentaries on the law of Municipal Corporations, Little, Brown and Co., Boston, 5. ${ }^{\text {a }}$ ed., 1911, vol. I, artículo 237. 
Con respecto al Gobierno Federal los Condados se encargan de la administración de temas agrarios, asistencia social y defensa civil (police power).

\section{La organización del Condado}

La estructura orgánica del Condado es bastante uniforme en todos los EE.UU., lo único que cambian son detalles puntuales en determinadas zonas de algunos Estados.

Juan José LAVILLA, en su estudio sobre el Condado en Inglaterra y Estados Unidos ${ }^{6}$, destaca tres rasgos comunes a los Condados de toda la superficie estadounidense:

Aparte de la heterogeneidad que ya hemos comentado, critica el arcaismo de la figura dada la inexistencia de un órgano ejecutivo unipersonal:

Estos entes no suelen contar con una persona encargada de las labores de dirección del Condado ${ }^{7}$, si bien existe la figura del «alcalde como director u organizador» con facultades administrativas que veremos más adelante cuando expliquemos los tipos de ayuntamientos en función del tipo de alcalde que exista. En Condados como Tennessee ${ }^{8}$ se puede encontrar en la actualidad reliquias del antiguo sistema condal inglés. En esta zona el gobierno y la administración del Condado están en manos de un grupo de personas a los que se le otorga la dirección de todo el Condado (justicies of the peace).

La tercera característica que, según LAVILla RUBiRA, define a los Condados es que suelen aparecer varios centros directivos, en un mismo Condado, dedicados a diferentes materias.

El organismo compuesto por el conjunto de esos departamentos encargados de las distintas materias del Condado recibe el nombre de Consejo

6 J. J. Lavilla Rubira, «El Condado de los Regímenes Locales Inglés y Estadounidense», pp. 593-634, dentro del estudio: La Provincia en el Sistema Constitucional, Diputación de Barcelona, varios autores, Madrid, 1991, pp. 624 y 625.

${ }^{7}$ Algunos Condados han adoptado el sistema de director en el que éste supervisa los diferentes departamentos. Pero este tipo de organización es muy escasa. Existe en Condados como Los Ángeles, San Diego o California.

${ }^{8}$ Tenn. Code Ann., Secc. 5-501-28. 
de Administración del Condado: este órgano es elegido bien por todo el Condado o por representantes provenientes de los Distritos en los que dicho Condado puede haberse dividido ${ }^{9}$. El número de miembros que lo compone puede ser muy dispar y oscila entre tres y más de setenta miembros. El poder que se da a esta administración le permite promulgar ordenanzas menores, pero su capacidad para legislar es pequeña ${ }^{10}$. Sus funciones son primordialmente administrativas: se encarga, sobre todo, de la administración de las finanzas y de las propiedades del Condado, de los contratos públicos y del mantenimiento de las carreteras. Si en el Condado no hay un departamento que se encargue de ello se debe ocupar de funciones sanitarias y de asistencia social y del funcionamiento de las elecciones en el Condado tanto locales como estatales o nacionales. También tiene como función la de aprobar el presupuesto anual y del nombramiento y supervisión de las labores de ciertos officers ${ }^{11}$ que trabajarán para el propio Consejo de Administración.

Todas estas labores corresponden al Consejo con carácter subsidiario, ya que, como se ha indicado antes, suelen ser realizadas por los diferentes departamentos. Como vemos, el poder del Consejo queda muy disminuido en la práctica y nunca podrá ejercer funciones de supervisión en los departamentos que, en la práctica, son los que asumen los pocos poderes que el Estado y la Nación delegan en estos entes.

El conjunto de officers que se encargan de los Departamentos son una prueba clara de la disparidad en la regulación en cada uno de los Estados y, dentro de éstos, de las diferencias de los Condados entre sí. En este sentido, cada Estado crea departamentos diferentes, que suelen coincidir con las necesidades de la zona que se encuentra bajo su dominio.

Los officers suelen ser elegidos de forma popular y son independientes tanto del Consejo de Administración del Condado como del resto de los Departamentos. La forma de elección viene dada por la Constitución del Estado al que pertenezcan. Sus funciones son meramente administrativas.

\footnotetext{
${ }^{9}$ En Connecticut y en Carolina del Sur los miembros son elegidos por el Legislativo del Estado.

${ }^{10}$ Esto supuso un logro jurisprudencial ya que anteriormente no se permitía a los Condados contar con funciones legislativas. V. Cogswell v. Sherman County, 238 Kan. 438 (1985).

${ }^{11}$ Este término se emplea para definir a todas las personas que trabajan para el Consejo de Administración y en los distintos departamentos. En este caso, como luego se aclara, nos referimos únicamente a los que prestan sus servicios en el Consejo.
} 
Veremos algunos de esos officers a continuación:

Los sheriffs ${ }^{12}$, policías y los abogados de la acusación ${ }^{13}$ se encargan de hacer cumplir las leyes dictadas por el Condado, así como de la administración de justicia.

Las labores en materias financieras se cumplen por officers encargados específicamente de esas funciones ${ }^{14}$. Éstos suelen ser elegidos por votación popular, si bien algunos autores lo critican ya que no tienen funciones políticas que cumplir y, por lo tanto, sería más lógico que fueran nombrados en lugar de ser elegidos.

Otros officers se encargan del registro. En la mayoría de los Estados esta función la realiza una persona elegida por votación popular y que es conocida con el nombre de Registrador del Condado. Suele, además, encargarse de las labores de Secretario en el Consejo de Administración del Condado y de Auditor del Condado.

Existen otros muchos officers que forman parte del personal al servicio de los departamentos de los Condados y que varían, como hemos indicado, de Estado a Estado en cuanto a su existencia, funciones o modos de ser elegidos ${ }^{15}$.

\section{Problemas de la figura del Condado: un futuro incierto}

Después de ver la disparidad de departamentos no puede extrañar que la figura del Condado, ya desde 1930, haya sido criticada por diferentes sectores. Autores como M. H. SATTERFIELD ${ }^{16}$ afirman que son entes lo-

\footnotetext{
12 Aparte de ser un funcionario encargado de mantener el orden también tiene tareas ejecutivas en los tribunales y es el encargado máximo de las cárceles del Condado. También es el encargado de dar licencias, recaudar toda clase de dinero de fianzas o multas de los delincuentes y participa en la elección de ciertos miembros de la Administración.

${ }^{13}$ Es el consejero del Consejo de Administración del Condado y de otros funcionarios en materias legales, redacta ordenanzas y contratos y actúa como fiscal en los tribunales persiguiendo las violaciones a la ley que se produjeron en la circunscripción del Condado o distrito. Representa al Condado y al Estado en las causas civiles de las que son parte.

${ }^{14}$ Auditor, asesor financiero, recaudador de impuestos, tesorero e interventor. Estas figuras no existen en todos los Condados.

15 Entre otros: Director de los Colegios del Condado, Directores del Consejo de Educación, Guardián de las Prisiones, Director de los Orfanatos del Condado, Director de Asuntos Sociales, Director de la Comisión de Planificación Urbanística o de la Comisión de Bibliotecas, Censor de Votantes, Inspector de Pesos y Medidas, Director de Sanidad, Abogado o Fiscal del Condado.

16 Vid. M. H. SATterfield, «Counties in a Strait-jacket», n. ${ }^{\circ} 37$, Revista Nacional Municipal, pp. 81-84 y 124, febrero de 1948 .
} 
cales que se han quedado atrás con respecto a Municipalities, Estados y Federación en la adopción de nuevas formas de organización, programas de personal y sistemas presupuestarios.

También se critica su descentralización en la dirección, ya que cada departamento funciona de forma independiente sin someterse a un director o miembro del ejecutivo que los supervise. Esto provoca que la responsabilidad se diluya al estar dividida en los diferentes departamentos.

La crítica también recae sobre los procedimientos que se utilizan, ya que muchos son de mero trámite y carecen de sentido en la actualidad y retrasan las actuaciones de los diferentes departamentos a la vez que dañan su efectividad. Por último, los autores se quejan de que no se apliquen métodos del sector privado en la organización interna, métodos que, en cambio, sí se emplean en las unidades de gobierno de las Municipalities o de los Estados para optimizar resultados ${ }^{17}$.

Otro fallo lo supone el elevado número de Condados que existen, muchos de ellos se conservan por reminiscencias históricas y hacen que a menudo haya una duplicidad de funciones con Municipalities y Estados.

Incluso la Liga Nacional de Municipios, ya en 1933, tachó a los Condados de «desmañados y chapuceros, anticuados e innecesariamente caros» ${ }^{18}$, la misma crítica se hizo en $1939{ }^{19}$ y los últimos estudios realizados confirman la continuidad de estos problemas incluso en la actualidad.

Como podemos ver por lo expuesto anteriormente, los Condados han sido criticados tanto por teóricos de la Administración Local como por la Liga Nacional Municipal o los propios ciudadanos que no ven con buenos ojos la doble imposición y la duplicidad de servicios que los Condados prestan para justificar, en muchos casos, el motivo de su existencia ${ }^{20}$.

17 Vid. James M. LANGLY, «Los Ciudadanos terminarían con los Condados», Revista Nacional Municipal, pp. 541-545, noviembre, 1948.

En la misma línea crítica se encuentran A. Strart, MacCorkle, D. WiLfred y WebB, que escribieron un artículo conjunto con el título de «Formas de Gobierno Local». Departamento de Investigaciones Municipales, Universidad de Texas, 1948.

18 Cfr. R. C. Atkinson, «Principios para un modelo de Gobierno de los Condados», Revista Nacional Municipal, n. ${ }^{\circ} 20$, pp. 469-486, septiembre de 1933.

19 Revista Nacional Municipal, n. ${ }^{\circ}$ 28, p. 78, septiembre de 1939.

${ }^{20}$ Un ejemplo de esta situación se produce en el Estado de Nueva York. La ciudad ha ido aumentando su radio de acción a zonas que antes se hallaban bajo el dominio del Condado por estar despobladas y no pertenecer a la ciudad. Estas zonas fueron incorporadas a la propia ciudad y en ellas los servicios son prestados por el gobierno del distrito al que pertenecen. El Condado continuó exis- 
Todas estas razones han hecho que parte de la doctrina y de los ciudadanos, así como las Municipalities, planteen la posibilidad de hacer desaparecer este tipo de entes locales. Los ciudadanos intentan evitar la doble imposición, sobre todo los de las ciudades. Pero a esto se oponen los que viven en zonas rurales en las que el Condado ve justificada su actuación. Las ciudades ven la desaparición de los Condados con deseos de obtener para sí los poderes otorgados a éstos. Y entre la doctrina no encontramos una posición clara.

Si nos fijamos en los autores de mediados de siglo podemos notar cómo entonces el Condado estaba en plena decadencia, dadas las críticas a las que se le sometía, como hemos visto antes. En algunos lugares el Condado desaparecía como tal y se unía a ciudades como sucedió en Filadelfia, Denver o Nueva Orleans. Autores de mediados de siglo, como. MACCORKLE o WEBB, interpretaban esta situación como muestras de la agonía que sufría la figura condal, que se iría debilitando para acabar desapareciendo absorbida por las grandes ciudades. Esta misma situación, en los cincuenta, se interpretaría de forma inversa: los Condados absorbían funciones propias de las ciudades y se creaban, así, los urban county, que llegarían al extremo de sustituir a la ciudad en la función de prestación de servicios ${ }^{21}$. Esto permitió a muchos autores, como ClaUNCH, reclamar un crecimiento de las competencias para los Condados ${ }^{22}$.

Desde finales de los sesenta la doctrina se dividía al respecto e incluso hubo autores ${ }^{23}$ que, conscientes de la necesidad de un cambio en la organización local, no conseguían decantarse por el modelo a seguir ni veían claro si debían entonar o no un requiem por una figura que en unos Estados llegó incluso a desaparecer ${ }^{24} \mathrm{y}$ que en otros aumentaba sus funciones e importancia ${ }^{25}$.

tiendo y prestando servicios que se entregan también por la ciudad a través del propio distrito de una forma más racional. Los ciudadanos se quejan de dicha situación y pretenden que el Condado desaparezca, cosa que no sucede por motivaciones políticas o de tradición histórica.

${ }^{21}$ En el Estado de Nueva York el Condado de Erie asumió las funciones de prestación de servicios en toda la ciudad de Búfalo.

22 Vid. ClaunCH, «Toward a More Effective County Government», Texas Law Review, vol. 35, 1957 , pp. 986 y ss.

${ }^{23}$ Vid. LINEBERRY, «Reforming Metropolitan Governance: Requiem or Reality», Georgetown Law Journal, vol. 58, 1970.

${ }^{24}$ Como es el caso de Connecticut.

${ }^{25}$ Vid. Comus, «Council of Government Approach», Vandervilt Law Review, vol. 22, 1969, pp. 811 y ss. 
Esta zozobra continúa hasta los noventa, pero a partir de este momento la práctica demuestra cómo otros entes, los Distritos Especiales, empiezan a proliferar a la vez que las Municipalities consiguen cada vez unos grados mayores de autonomía.

Las competencias de los Condados se reducen de forma considerable en favor de las municipalites y, sobre todo, de los Distritos Especiales que a lo largo de la última década han demostrado la eficacia de su actuación y han reducido las esperanzas albergadas por los nostálgicos defensores de la antigua institución inglesa.

\section{ENTES CREADOS CON UNA FINALIDAD CONCRETA: ESPECIAL REFERENCIA A LOS DISTRITOS ESPECIALES}

Debemos a William G. ColmaN ${ }^{26}$ la distinción entre dos grupos de entes locales: entidades locales de carácter general (Condados y Municipalities) y Special-purpose Governments, que son los que vamos a explicar a continuación.

Antes de pasar a explicar las funciones que ostentan las Municipalities en el régimen local estadounidense queremos centrar nuestra atención en los Distritos Especiales que, como hemos dicho, han proliferado en la última década absorbiendo funciones tanto de Condados como de las propias Municipalities (ciudades y townships). De una forma menos importante y numerosa, algo parecido ha sucedido con otro tipo de unidades locales de menor entidad que también analizaremos en segundo lugar.

\section{Los Distritos Especiales}

\subsection{Concepto}

La mayoría de los autores coinciden al definir a los Distritos Especiales como entidades locales independientes ${ }^{27}$ creadas ad hoc ${ }^{28}$ por leyes que emanan de la cámara legislativa del Estado o por normas federales.

\footnotetext{
${ }^{26}$ Vid. G. W. Colman, State and Local Government and Public-private Partnerships, Connecticut, 1989 , pp. 19 a 59.

${ }^{27}$ Cfr. N. BuRns, The Formation of American Local Governments: private values in public institutions, Nueva York, 1994, p. 4.

28 Vid. William Vemon Holloway, American Government, National, State and Local, cap. 25, Nueva York, 1959.
} 
Estas normas les suelen dotar de poderes impositivos, poderes para perseguir deudas y poder de expropiación.

Generalmente funcionan para ofrecer un tipo de servicio concreto o, como mucho, un conjunto de servicios relacionados entre sí ${ }^{29}$. Su área de actuación es ilimitada, algunos la extienden sólo dentro de las ciudades y otros, incluso, despliegan sus funciones en zonas que superan las dimensiones de los Condados.

Como hemos aclarado, este tipo de entes ha proliferado mucho en la última década. En la actualidad existen casi treinta mil Distritos Especiales en los Estados Unidos ${ }^{30}$.

Pese a que los Distritos Especiales aparecieran por vez primera hace doscientos años, hay que aclarar que su auge comenzó a notarse desde $1920^{31}$. La mayor parte de los distritos que se crearon desde este momento eran distritos encargados de labores de reparto de agua o de energía y todos estaban relacionados y se creaban por los Departamentos de agricultura de los Estados, con el fin de ser más eficaces a la hora de solucionar problemas en zonas rurales. Su proliferación desenfrenada ha hecho que desde ese momento hasta la actualidad su número haya aumentado, al igual que las funciones para las que son creados.

Este incremento no ha sido regular en todos los Estados. Aparte de la tradición histórica otros son los factores que pueden determinar, según William G. Colman, que en unos Estados estos entes hayan proliferado más que en otros, como son los poderes con los que cuentan los entes superiores (Condados, ciudades o el propio Estado) o la existencia o no de grandes distancias entre las diferentes concentraciones urbanas (que hará que estos distritos sean más necesarios).

\subsection{Organización de los Distritos Especiales}

Dejando a un lado las razones de su aparición veamos su utilidad y los medios que los distritos especiales emplean para prestar sus servicios.

\footnotetext{
29 Vid. William G. Colman, State and Local Government and Public-Private Partnerships, Greenwood, 1989, p. 22. Como ejemplo podriamos poner los distritos de agua y alcantarillado que se ocuparán de todo lo referente al agua que se consume y utiliza en una zona concreta.

30 Vid. D. L. Chicone y N. WaLzer, Governamental Structure and Local Public Finance, New York, 1985, pp. 8 a 16.

31 Vid. E. S. GRIFFITH, A History of American City Government: the progressive years and their aftermath, 1900-1920, Washington, 1983, p. 298.
} 
Los Distritos Especiales se ordenan de modo diferente al de las demás entidades locales, que siguen criterios de división territorial. Esto hace que el ciudadano se encuentre confuso, pues, perteneciendo a una misma ciudad, los servicios que obtienen pueden ser prestados por diferentes entes: la misma ciudad, el Condado, el Estado, una Agencia Federal ${ }^{32}$ o varios Distritos Especiales. En muchos casos el área de actuación de estos últimos es desconocido incluso por los miembros de gobierno y funcionarios de los ayuntamientos ${ }^{33}$.

La mayoría de los Distritos Especiales tiene un Consejo de dirección que se encarga de dar una unidad a las decisiones y medidas a adoptar para la prestación del servicio. En los distritos que se crean en la actualidad la figura del secretario o administrador se hace indispensable para supervisar el trabajo contable y más complejo del distrito.

La elección de los miembros de dichos entes locales es también muy diferente a la que se exige en las Municipalities. El Tribunal Supremo declaró que no tenía por qué seguirse el principio de un hombre un voto ${ }^{34}$. Esto llevó a que en la práctica se haya restringido la participación del ciudadano en las elecciones de officers de los Distritos Especiales y que se permita el voto a no residentes que tienen intereses o propiedades en la zona ${ }^{35}$.

Muchos de los miembros que trabajan en los Distritos Especiales no son ni siquiera elegidos sino nombrados. En muchos casos estos nombramientos no tienen en cuenta la decisión de los ciudadanos.

Esta forma de gobierno ha sido duramente criticada tanto por grupos de ciudadanos, que ven afectados sus intereses, como por la doctrina que duda de la constitucionalidad de los comicios y plantea la necesidad de

\footnotetext{
32 Vid. E. Carbonell y J. L. Muga, Agencias y procedimiento administrativo en Estados Unidos de América, Madrid, 1996. También E. GARCIA DE ENTERRIA, «Algunas reflexiones sobre el Derecho Administrativo norteamericano (a propósito de la nueva ordenación sistemática del mismo)», RAP, núm. 85, 1978. Y A. M. MORENO MolNA, La Administración por Agencias en los Estados Unidos de Norteamérica, Madrid, 1995.

${ }^{33}$ Cfr. N. Burns, The Formation of America Local Governments, Oxford University Press, New York, 1994, p. 14.

${ }^{34}$ Caso Salyer Land Co. v. Tulare Lake Basing Water Storage District, 410, US 719 (1973). Dice que en las elecciones sólo podrán participar terratenientes.

35 Vid. Richard BriffaurT, «Who Rules at home?: one person one vote and local Governments», University of Chicago Law Review, . $^{\circ} 60$, Spring, 1993. Explica el origen de esta restricción del derecho de sufragio. Éste se encuentra en los water districts que permitían el voto sólo a los terratenientes, más conocido como landowner voting.
} 
una reinterpretación jurisprudencial del concepto de derecho activo de voto ${ }^{36}$, como en tímidas ocasiones ha hecho la jurisprudencia ${ }^{37}$.

\section{Otros organismos con finalidades específicas}

\subsection{Distritos Escolares Dependientes}

Esta figura es la más frecuente, numerosa e importante de las que vamos a tratar. Los calificamos como dependientes porque la mayoría de estos distritos actúan como una extensión y dentro del territorio de las Municipalities (ciudades, pueblos) o de los Condados, si bien, como dijimos en un principio, su creación o extinción no depende del deseo de éstos sino de entidades con más poder como el Estado y, en su caso, la $\mathrm{Fe}$ deración. Esta subordinación no tiene otro fin sino el de conseguir unas prestaciones mejores evitando solapamientos y comportamientos opuestos que entorpezcan las labores de uno y otro tipo de entes en perjuicio de los ciudadanos.

En los diferentes Estados el trato dado a la figura es distinto, dependiendo de quien tenga atribuidos los poderes en materia educativa. $\mathrm{Si}$ bien su existencia está generalizada, como dato curioso podemos destacar que en Alaska, Hawai, Maryland, Carolina del Norte y Virginia no hay Distritos Escolares Dependientes. Esto se debe a razones de tipo fundamentalmente impositivo. En estos lugares todas las escuelas públicas se administran por medio de las agencias del Condado, ciudad o del propio Estado.

\subsection{Areas impositivas subordinadas a los Condados}

Pese a no ser unidades de gobierno local, están autorizadas legalmente para prestar un servicio en concreto sin necesidad de crear un distrito específico. Los gastos que conlleva la creación de estos entes se cubren mediante impuestos adicionales que afectan a aquellas personas que se benefician del servicio prestado por estas entidades en función del aumento

36 Vid. R. T. ForD, «Beyond Borders: a partial response to Richard Briffault», Stanford Law Review, mayo, 1996, pp. 1173 a 1195. En este artículo se hace un resumen del Symposium que se celebró en este año para discutir la posibilidad de ampliar el derecho de voto a personas que, no viviendo en un ente local en concreto, utilizan sus servicios o se ven afectados por una entidad local cercana a la suya. Se pensó que se debería permitir a éstos participar en los comicios.

37 Vid. Hadley v. Junior College District of Metropolitan Kansas City, 397 US 50, 1970. 
del valor del terreno o bien inmueble que se experimenta tras la incorporación de dicho servicio.

\subsection{Las asociaciones de vecinos o ciudadanos}

Las neigborhood subunits y citizen associations ${ }^{38}$ tampoco están recogidas en los censos de entidades locales si bien son esenciales en el desarrollo y funcionamiento local. Se encuentran unidas a los entes locales en muchas áreas urbanas. Ciudades como Indianapolis, Washington, Nueva York, San Pablo o Boston han tenido en algún momento en las dos últimas décadas alguna de estas formas de gobierno que han tomado el nombre de gobierno de la ciudad o «neighborhood city halls» o han surgido como una mezcla de ambos entes. Numerosas ciudades han utilizado este tipo de entidades con el fin de descentralizar algunos de los servicios sociales y sanitarios que en un principio eran prestados por aquéllas. La idea: crear centros de multiservicios que se ofrecen a los ciudadanos de una determinada zona con unas determinadas necesidades en concreto.

Estas asociaciones cada día toman una mayor importancia en las ciudades tanto desde el punto de vista de la prestación de los propios servicios como desde el punto de vista político. Estos entes participan en el proceso legislativo y su influencia se acentúa cada vez más pese a tener una función meramente consultiva en el proceso legislativo.

Muchos autores que siguen la corriente de MARTIN ${ }^{39}$ encuentran en este tipo de entes la reacción silenciosa al tan criticado fracaso electoral que sufre el país tanto en la elección de Presidente como a la hora de nombrar a los más directos representantes del ciudadano en los Ayuntamientos. Muchas de las actuaciones administrativas de los ayuntamientos son técnicas y no llegan a los oídos del electorado a no ser por las deficiencias en los servicios que se manifiestan cuando la crisis y la corrupción en el ayuntamiento están demasiado avanzadas. La solución que se ha tomado en numerosas ciudades ha sido la de descentralizar la administración en lo que popularmente se ha llamado "gobiernos de barrio" o "pequeño Ayuntamiento" ${ }^{40}$. Las ventajas que este sistema plantea son las respuestas concretas a las necesidades de cada zona, la mejora en la

\footnotetext{
38 Vid. H. Hallman, Neighborhodds: Their Place in Urban Life, Califormina, 1984.

39 David L. MARTIN, Running a City Hall Municipal Administration in America, Alabama, 1990.

40 Vid. P. Epstern, «How Citizen Participation Spruces Up Performance», National Civic Review, marzo-abril, 1987, pp. 147 a 151.
} 
prestación del servicio y el mayor alejamiento del juego político, a la vez que se produce un acercamiento al ciudadano.

Esta creciente importancia ha hecho que desde ciertos sectores (ACIR) se haya solicitado a los Estados que creen una legislación para autorizar la fundación de este tipo de entes u otros menos formales compuestos por la asociación de diferentes vecinos. La iniciativa para su creación debe partir de los propios vecinos o del equipo de gobierno de la ciudad o del Condado interesado ${ }^{41}$.

\subsection{Asociaciones de condominio (vecinos), caseros y cooperativas}

Suponen, al igual que los entes anteriores, una importante ayuda para los gobiernos locales en ciertas áreas urbanas. Pese a su simplicidad y espontaneidad, las comunidades de vecinos complementan e incluso reemplazan ciertos servicios municipales. En algunos aspectos constituyen auténticas formas privadas de gobierno ${ }^{42}$.

Como resultado de su crecimiento e importancia en 1973 se creó el Instituto de Asociación de Comunidades, al servicio de todas estas comunidades ${ }^{43}$.

\subsection{Organizaciones para la planificación y coordinación de áreas metropolitanas y otras áreas en el ámbito inferior al Estado}

En un principio se crearon por el Estado federal para la revisión de las relaciones entre los diferentes entes locales y para detectar su eficacia y el cumplimiento de ciertos objetivos. Pero ahora funcionan para coordinar acciones entre los distintos Condados y otros entes locales bajo la circunscripción de un Estado en concreto.

David WALKER ${ }^{44}$ ha identificado 17 formas de cooperación entre los distintos entes locales, que van desde la mera función consultiva por par-

41 Vid. G. W. LiebmanN, «Devolution of power to community and block associations», Urban Lawyer, 1993.

${ }^{42}$ K. Rosenbery, «Condominium and Homeowner Associations. Should they be Treated like Mini-Governments?», Zoning and Planing Report, octubre 1985, pp. 153-158.

43 Vid. Instituto de Asociación de Comunidades, Publications Catalog, 1988-89, octubre de 1987.

${ }^{44}$ Cfr. D. WALKER, «Snow White and the 17 Dwarfs: from metro cooperation to governance», $\mathrm{Na}$ tional Civic Review, enero-febrero, 1987, pp. 14 a 28. 
te de los gobernantes de los diferentes Condados o Municipios hasta la acción conjunta a la hora de ofrecer un servicio determinado.

En muchas áreas metropolitanas estos tipos de gobierno toman la forma de «Councils of Governments» COGs, compuestos por representantes elegidos en cada una de las zonas a las que luego representan. Las funciones que cumplen son de intercambio de información en problemas comunes; acuerdos para la solución de conflictos; recolectar la información de las diferentes áreas en cuanto a las cuestiones que los ciudadanos plantean.

Todas estas asociaciones encuentran un punto de apoyo y ayuda en la Asociación Nacional de Consejos Regionales (NARC) que ofrece asistencia técnica y publicaciones a las asociaciones afiliadas.

\section{Críticas}

Para terminar, debe destacarse que la formación de esos distritos especiales ha sido una materia controvertida y criticada por los officers de otros entes locales ante el miedo de perder competencias y la consiguiente financiación y no así por los propios ciudadanos que ven cómo estos entes estimulan la participación en los asuntos que les afectan de forma directa.

La crítica se centra en dos planos diferentes:

La posible duplicidad en la prestación de servicios y en la persecución de fines idénticos por parte de estos entes que se suelen superponer a los entes locales. Muchas veces con acierto, puesto que muchas funciones que son competencia de los entes locales tienen unos costes excesivos o los servicios que se deben prestar no se prestan en debida forma o, incluso en determinadas circunstancias, no llegaron a ser recibidos por los ciudadanos. A menudo, los Distritos Especiales pueden ofrecer servicios de un modo mucho más eficiente, ya que el área en la que prestan dicho servicio es más pequeña y permite una mejor financiación. Por ejemplo, se pueden establecer diferentes grupos de sujetos pasivos a la hora de recaudar impuestos para financiar esos servicios al igual que es más fácil llegar a conocer las preferencias de los consumidores de dicho servicio ${ }^{45}$.

45 Vid. P. Downing y T. Di LoRenzo, User charges and Special Districts, Washington DC, 1987, pp. 277-284. 
Si bien estas críticas pueden ser rebatidas desde diferentes puntos de vista, creemos que el más acertado es el que resalta la importancia de estos distritos especiales a la hora de permitir la participación cívica y política de los ciudadanos en las unidades de gobierno. James MiCHENER explicó este punto de vista a principios de los sesenta en una reunión de parlamentarios estatales en Philadelphia. Dada la situación actual en los EE.UU. de total desinterés por el gobierno a cualquier nivel y la escasa participación en las elecciones, estas oportunidades de participación deben ser tomadas en cuenta a la hora de reorganizar el gobierno local ${ }^{46}$.

\section{MUNICIPALITIES}

\section{Concepto}

Son entidades locales creadas por los Estados bajo el consentimiento de la población que esté dentro de su territorio de acción mediante la figura del referéndum. Están dotadas de autonomía para la gestión de sus intereses propios ${ }^{47}$ y satisfacción de las necesidades de la comunidad local. Esta autonomía hace que, al contrario que los Condados ${ }^{48}$, puedan incurrir en responsabilidades. Las Municipalities despliegan sus poderes en una zona determinada e independiente con respecto a entidades locales superiores. Las competencias son delegadas por el Estado mediante la constitución o normas dictadas por la Cámara legislativa del Estado.

La clasificación más tradicional de las municipalities las diferencia atendiendo a su tamaño en: townships, ciudades (cities), pueblos (towns) o villas (villages) ${ }^{49}$. Dado que las tres últimas sólo se diferencian por el número de habitantes, hemos considerado de mayor interés las dos primeras que pasamos a desarrollar.

\footnotetext{
46 Vid. W. Miller, «American National election Study», National Civic Review, mayo-junio de 1987, p. 193.

${ }^{47}$ Conocido como local self-government que debe ser reconocido de forma expresa por el Estado mediante statutory o constitutional home rule.

48 Caso Champlim Petroleum Co. v. Board of County Commissioners, 526 P. 2d 1142, OK (1974). En este caso se alega que los Condados cuentan con sovereign inmunity.

49 Vid. David L. MARTIN, Running a City Hall Municipal Administration in America, Alabama, 1990, p. 1.
} 


\section{Townships}

Legalmente se les considera como quasi-corporaciones y son subdivisiones que se efectúan dentro del territorio del Condado para la prestación de servicios y organización de zonas rurales. No suelen ejercer ningún tipo de jurisdicción sobre los municipios. Como quasi-corporaciones que son pueden ser titulares de bienes, contratar, demandar y ser demandados, si bien su responsabilidad no es tan amplia como en el caso de las ciudades, precisamente por la función ejecutiva que cumplen estas últimas en el ámbito local.

Si bien nos hemos aventurado a dar una definición general de estos entes las características de cada uno de ellos son muy diferentes entre los Estados. Incluso el nombre que reciben es distinto: towns, en los Estados de Nueva Inglaterra; plantations en Maine; locations en Nueva Hampshire.

Hay dos tipos de townships. Unos reciben el nombre de congressional townships, que no constan de un órgano local central para la toma de decisiones y que sólo se encargan de llevar un registro de los títulos de propiedad de las tierras y actúan sin independencia bajo la supervisión de las Agencias federales. Esta clase de township no es un ente local al carecer de independencia. Por eso no nos extenderemos más en su explicación y nos centraremos en el otro tipo de township al que se conoce con el nombre de civil.

Sus funciones varían, como hemos dicho, dependiendo del Estado en el que nos encontremos. Las más comunes son las de mantenimiento de las carreteras, asesorar al Estado en materia de impuestos, expender licencias, administrar programas de asistencia social y participar en la aplicación de las normas mediante su propia policía. También deben encargarse de la construcción y dirección de parques, bibliotecas y hospitales. En algunos Estados tienen asignadas responsabilidades para la administración escolar.

Otra característica de las municipalidades es que delimitan el territorio en el que los jueces de paz deben ejercer su jurisdicción en asuntos civiles y penales menores en los juzgados de paz.

Nos gustaría destacar las peculiaridades de esta forma de entidades locales en el tercio norte de los EE.UU. En esta zona el municipio es un ejemplo directo de democracia participativa: en las reuniones los votantes de la zona participan y discuten los asuntos, eligen a los officers, se votan los impuestos y se aprueban las adquisiciones de bienes del ente. 
Como comprobaremos más adelante, su parecido con los municipios menores o pueblos de Nueva Inglaterra es enorme, si bien estos últimos tienen mayores libertades.

Esta forma de gobierno tuvo mayor importancia en los inicios de la historia de los EE.UU., en esta época la población se agrupaba en las zonas rurales y la escasez en los medios de transporte hacía que la vida rural estuviera más consolidada.

En la actualidad esta forma de gobierno se encuentra en declive y su número ha disminuido en gran cantidad de Estados y sus funciones han sido traspasadas a los Condados y a los Estados ${ }^{50}$.

\section{La ciudad}

Existen en los 50 Estados y son las unidades más importantes del Gobierno Local. Abarcan menos territorio que las anteriores pero ejercen más poderes y proveen una mayor variedad de servicios que el Condado. El número de municipios no aumenta de modo importante si lo comparamos con el tremendo desarrollo sufrido por los Distritos Especiales en los últimos años.

La cantidad de ciudades con las que un Estado cuenta varía mucho a lo largo de la geografia estadounidense y depende fundamentalmente de las normas de incorporación, esto es, de los criterios que deben seguir las ciudades para su formación. Por ejemplo, el Estado de Illinois tiene más de 1.200 municipios. En cambio, Hawai y el Distrito de Columbia -que alberga a la capital de la nación - sólo guardan dentro de su territorio una ciudad ${ }^{51}$. Otro de los criterios que marca diferencias, como hemos venido indicando, es la influencia que tuvo la ciudad a lo largo de la historia.

Es importante destacar las reducidas dimensiones de los municipios en los EE.UU., la mitad de éstos tienen una población que no supera los 1.000 habitantes. Lo cual justifica el elevado número de casos en los que los entes locales actúan de forma espontánea, desoyendo las normas existentes 52 .

\footnotetext{
so Vid. Clyde F. SNider, «The Twilight of the Township», n. ${ }^{\circ} 41$, Municipal Review, pp. 390-396, septiembre de 1952; y también en la obra «Townships Lose Ground», n. ${ }^{\circ} 41$, Municipal Review, pp. 10-13, enero de 1955.

${ }^{51}$ Vid. W. V. Holloway, American Government, National, State and Local, cap. 22, Nueva York, 1959, pp. 371 y ss.

s2 Vid. «Governmental organization», Census of Governments, vol. I, Washington, 1988.
} 
Para explicar este tipo de ente local vamos a partir de las características propias de la ciudad que anteriormente hemos citado.

\subsection{El nacimiento del municipio}

"Las ciudades son entidades locales creadas por los Estados bajo el consentimiento de la población que esté dentro de su territorio de acción mediante la figura del referéndum.»

Desde 1868 se asentó por la jurisprudencia el principio de subordinación de los entes locales al Estado. Entre otras atribuciones el juez DiLlON otorgó a los Estados la posibilidad de crearlos y destruirlos 53 . Para él las ciudades eran «... meros entes creados por los Estados a los que deben su nacimiento» ${ }^{54}$. Esta teoría recibió numerosas críticas y no se aplicó por todos los tribunales ${ }^{55}$, pero, pese a esto, sigue siendo la doctrina tradicional que se aplica por la mayoría de los tribunales ${ }^{56}$.

Para analizar el problema de la creación de la ciudad podemos partir de las palabras de la Corte Suprema de Florida en 1933: «... son dos los elementos esenciales para la existencia de un municipio; una comunidad humana y el territorio que ésta ocupa. La Cámara legislativa del Estado no puede crear ninguno de estos dos componentes» ${ }^{57}$.

Mediante el «Acta General sobre Formación de Municipios» el legislativo del Estado de Florida, al igual que en el resto de los Estados ${ }^{58}$, especifica las condiciones para la creación de una ciudad (incorporation).

Se exige un número mínimo de habitantes, un número de votantes registrados o un mínimo de densidad de población por millas cuadradas. El requerimiento se plantea ante un tribunal, asociación del Condado, o cualquier otra agencia local con una comisión de formación designada por el Estado para emitir órdenes de incorporación.

53 Caso City of Clinton v. Cedar Rapids and Missouri Railroad Company, 24 Iowa (1868).

54 J. F. Dillon, Commentaries on the Law of Municipal Corporations, 5. a ed., Boston, 1911, vol. 1, p. 448. «Municipal Corporations owe their origen to, and derive their powers from the state legislature. It breathes into them the breath of life, without which they cannot exist.»

55 Caso People v. Hurlbut, 24 Mich. 44 (1871).

56 Vid. 56 Am Jur 2d, Municipal Corporations, Counties, and other Political Subdivisions, p. 28.

57 Caso State of Florida ex rel. Davis att. gen. v. Town of Lake Placid, 109 Fla. 419 (1933).

58 Vid. 25 NY Jur 2d, Incorporation of Villages, p. 12. 
El municipio que se propone debe ocupar un área de terreno concreto, si bien no es necesario que ésta sea muy grande. En algunos Estados se exige que la solicitud de incorporación vaya firmada por un cierto número de dueños del terreno circunscrito en el futuro municipio, cuyas propiedades deben suponer un porcentaje de los terrenos de ese futuro ente. Ésta ha sido la causa de que algunos municipios tengan una forma peculiar dado que los terratenientes se negaron a que sus tierras se incorporasen a esa zona.

La petición va dirigida a un tribunal que se encarga de revisar si se cumplen las condiciones que el Estado pide para dicha incorporación. En algunos casos estas decisiones se realizan bajo la forma de audiencia pública. Si las citadas condiciones se cumplen se realiza un referéndum entre los ciudadanos del futuro municipio. La mayoría de los votos emitidos por los vecinos del futuro municipio deben ser favorables a la incorporation, en este caso el juez del tribunal o el funcionario responsable (en el caso de que la decisión fuera tomada por una asamblea creada al efecto) dictará una orden declarando que el municipio se ha creado. En las State General Municipal Acts, aparte de los criterios para la formación, se clasifican los entes para su mejor organización ${ }^{59}$.

A continuación vamos a ver las formas que tiene el municipio para poder ampliar sus límites una vez que se ha creado. Estos medios tienen que estar regulados expresamente por la Carta de la Ciudad dada por el Estado. La anexión y la ampliación de poderes son las dos formas que permiten a la ciudad extender su ámbito de actuación.

Mediante la anexión la ciudad extiende sus límites y poderes de actuación a territorios que no se incorporaron a ella cuando se formó y que tampoco pertenecen a otra ciudad (town o village).

En algunos Estados la anexión requiere un acto por parte de la Cámara Legislativa de éste. En cambio, en otros es suficiente con la revisión de la propuesta por un tribunal o una comisión encargada de ello. A este requisito se añade el del referéndum ${ }^{60}$, en el que se exige la mayoría simple de votos a favor. El derecho de sufragio recae tanto en los habitantes de la

\footnotetext{
59 En ellas se clasifican los distintos municipios atendiendo a criterios de extensión, población, recursos económicos... todos estos parámetros se utilizarán posteriormente para saber qué política impositiva se les debe aplicar, los servicios que deben ofrecer o la forma de gobierno más correcta a adoptar para que consigan una mejor gestión. Vid. David L. MARTtN, Running a City Hall Municipal Administration in America, Alabama, 1990, pp. 20 y 21.
}

${ }^{60}$ Vid. 25 NY Jur 2d, Annexation, pp. 43-61. 
ciudad como en los del territorio que se va a anexionar. En algunos casos el derecho de voto se restringe a los dueños de las tierras que se van a anexionar.

Como hemos visto, la anexión es un acto bilateral si bien caben algunas excepciones, como en el caso de que el área que se anexiona se encuentre deshabitada (o tenga muy poca población). En este caso basta con el voto favorable de los habitantes de la ciudad, sin necesidad de que se proceda a la votación por parte de los habitantes del territorio.

Las principales consecuencias de la anexión son las políticas. Es por este motivo por el que, en la mayoría de los casos, el área que se anexiona se niega a tal proceso. Las razones que provocan esta reticencia son, generalmente, el miedo al incremento de los impuestos y la condición de los propios habitantes de estas zonas: personas que en su momento decidieron escapar de la ciudad, de los problemas de ésta y los elevados precios. Para combatir esta postura las ciudades ofrecen servicios a los futuros ciudadanos.

Se conoce por poderes extraterritoriales los que la ciudad tiene y utiliza más allá de sus propias fronteras. Describiremos alguno de los casos más frecuentes:

El primero de ellos se produce como consecuencia de la condición de propietarios que ostentan las ciudades fuera de sus límites como, por ejemplo, los aeropuertos ${ }^{61}$.

Otro ejemplo es el derecho a cobrar impuestos a todas las personas que trabajan dentro de la ciudad, aunque no vivan en ella ${ }^{62}$.

En otros lugares la ciudad se reserva el derecho de exigir licencias para el ejercicio y apertura de ciertos negocios en zonas cercanas a la ciudad. Estas zonas reciben el nombre de «jurisdicciones de policía» (police jurisdiction).

El caso más extremo es el que se produce en ciertos Estados en los que se permite a la ciudad el control sobre la planificación y la división de los terrenos y del uso de la tierra fuera de sus propios límites.

61 Vid. 27 NY Jur 2d, «Port Authority of New York and New Jersey», pp. 1192-1206.

62 Decisión que se tomó en ciudades como Washington o Nueva York tras la masiva huida de los trabajadores a zonas adyacentes (Virginia o Nueva Yersey), para evitar los elevados impuestos. 


\subsection{La muerte del municipio}

Los municipios gozan del derecho de existencia a través del tiempo. Una vez que el Estado ha creado un municipio éste no puede eliminarlo. Éste es el resultado de una interpretación amplia de la teoría creada por DILlON y que tiene su origen en la práctica cotidiana de los Estados y en alguna sentencia ${ }^{63}$. A los candidatos electos en un Estado no les interesan los resultados políticos que podría tener terminar con un municipio. En la actualidad la única reminiscencia que queda del poder de los Estados para terminar con los municipios es la necesidad de que la Cámara Legislativa así lo declare, si bien el proceso por el que se toma esa decisión está en manos de los habitantes de dicho ente ${ }^{64}$. Veremos a continuación las formas frecuentes por las que un municipio puede desaparecer:

La ciudad puede decidir, mediante una votación, su propia disolución si los contribuyentes opinan que los servicios son muy caros y que podrían mejorarse si fueran prestados por otro ente en un nivel diferente dentro de la escala territorial.

La decisión se puede tomar también por considerarse más favorable para sus intereses cívicos. Esto suele producirse ante la proclamación de nuevas normas restrictivas de derechos, como prohibición de determinados juegos, restricción de horarios de apertura de establecimientos al público..., etc.

Otra causa del cese de la existencia de un municipio se manifiesta claramente en el fenómeno de los pueblos fantasma. En estos casos los entes han dejado de ejercer los poderes propios concedidos por el Estado como recaudación de impuestos, elección de concejales y otras muchas funciones vitales para el mantenimiento físico del municipio. En estas circunstancias el pueblo se extingue legalmente si transcurre un determinado período de tiempo. Para que el municipio deje de existir es necesario que la legislatura del Estado así lo declare. Si bien, en la mayoría de los casos basta con la declaración de extinción por parte del Abogado del Estado.

${ }^{63}$ Caso People v. Hurlbut, 24 Mich. 44, (1871). «Local Government is a matter of absolute right, and the State cannot take it away».

6456 Am Jur 2d, Municipal Corporations, Counties, and other Political Subdivisions, pp. 89-90. 


\subsection{Delegación de poderes}

En la definición que estamos desarrollando se decía que las ciudades:

«... están dotadas de autonomia para la gestión de sus intereses propios (self-government) y satisfacción de las necesidades de la comunidad local [...], las competencias son delegadas por el Estado mediante la constitución o normas dictadas por la Cámara Legislativa del Estado.»

El derecho de autonomía para la gestión de los intereses propios debe ser conferido de forma expresa por el Estado. Este derecho no es común a todas las ciudades de los Estados Unidos sino propio de aquellas que gozaban de él antes de que se aprobase la Constitución ${ }^{65}$. En cualquir caso, el derecho de home rule está siempre limitado por los poderes del Estado. La delimitación de poderes se hace siguiendo la regla de DiLlon, que previamente explicaremos.

\subsubsection{La regla de DiLlon}

La revolución norteamericana liberó a las 13 colonias y las transformó en Estados, fenómeno que tuvo un efecto negativo sobre la autonomía de la que habían disfrutado las ciudades. Antes de la revolución, y de pasar del control de los Gobernadores Coloniales ingleses al del Poder legislativo de los Estados, las ciudades tenían una total capacidad de autogobierno: ellas decidían sobre su propia creación y las normas que regularían su funcionamiento posterior. La ciudad gozaba de total independencia, como Jefferson defendía ${ }^{66}$.

Tras la revolución los Estados ganaron poderes que les permitían cambiar las cartas otorgadas a los municipios años atrás e incluso disolverlos. Pese a esto, los Estados evitaban intervenir excesivamente en los asuntos locales.

La industrialización produjo grandes cambios en las relaciones entre el Estado y las ciudades. Después de la Guerra Civil las ciudades comenzaron un proceso de expansión que se vio afectado por la emigración de multitud de familias que se trasladaron del campo a la ciudad o que venían de Europa para trabajar en las nuevas industrias.

En este clima industrial eran los dueños de las fábricas los que ostentaban el poder político. Éstos eran los que designaban a las personas que

${ }^{65}$ Cifr. 56 Am Jur 2d, Municipal Corporations, Counties, and other Political Subdivisions, p. 125.

66 Vid. Syed ANwar, Political Theory in American Local Government Law, Nueva York, 1966. 
debían presentarse en cada departamento dentro del Ayuntamiento. Para controlar los votos hacían uso del poder económico que tenían sobre sus empleados ${ }^{67}$. Se puede afirmar de modo contundente, como han hecho numerosos autores ${ }^{68}$, que esta época ha sido la de mayor corrupción de las ciudades en los Estados Unidos ${ }^{69}$. Como afirma D. L. MARTín ${ }^{70}$, la evidencia de la corrupción se puede percibir en los edificios que albergaron a aquellos officers corruptos. Los Ayuntamientos tomaron la apariencia de palacios, cuya construcción servía de negocio para los propios alcaldes que eran los dueños de las empresas constructoras a las que se les encargaba la ejecución de dichas obras.

La corrupción empezó a ser criticada desde todos los niveles políticos y sociales.

Como ejemplo podemos recordar las palabras de Lord Bryce, embajador de Gran Bretaña en EE.UU., que en 1888 escribió: «los Gobiernos Locales son la muestra más obvia del fracaso de la Democracia Americana» ${ }^{71}$.

Desde la prensa también se criticó rotundamente este fenómeno. Lincoln STEFFENS ${ }^{72}$, al hablar del fenómeno de la corrupción, utilizaba palabras como: soborno, derroche, chanchullos y manipulación de las elecciones.

A principios de 1880 algunos políticos fueron elegidos para que impulsaran el cambio de los gobiernos municipales. Un ejemplo de alcalde reformista lo tenemos en Grover Cleveland que se hizo oír en la Casa Blanca junto a otros alcaldes que crearon la llamada «Liga Nacional Municipal» que desde 1894 se propuso dar soluciones que duran hasta hoy día y mejoraron la situación.

67 Vid. J. F. Richardson, «Del periodo colonial a 1901», en la revista New York Policy, 1970. O J.

C. Viterittr, Policy, 1995, pp. 910 a 912.

68 Entre otros tiene relevancia Robert A. DAHL, “QQuién gobierna?», Revista Democracia y poder en una ciudad americana, pp. 11 a 85 (1961).

69 «Community and american federalism: images romantic and real», Joseph P. VITERTTI y Gerard J. Russello, Revista de Virginia de Politica Social y Derecho, primavera 1997, p. 702.

70 Running City Hall. Municipal Administration in America, Ed. Universidad de Alabama, 1990, 2. edición, pp. 3 a 6.

71 El Estado del Bienestar en América.

72 La vergüenza de las ciudades, 1904. 
La mayoría de los alcaldes corruptos desaparecieron tras la II Guerra Mundial. Si bien no se pueden olvidar figuras como la del alcalde Richard J. Daley, al que se le conoció con el nombre de «el último jefe» (the last boss). Lo único que pudo acabar con su alcaldía fue su propia muerte que le sobrevino en el despacho de su ayuntamiento en 1976. Pero esto no fue lo que más sorprendió a la opinión pública y a los medios de comunicación del resto del país. La elección de su propio hijo como alcalde en 1989 confirmó el dicho de que «Chicago todavía no estaba preparada para una reforma» y demostraron que los rumores sobre el caciquismo eran ciertos ${ }^{73}$.

Ante esta situación la jurisprudencia, apoyada por la opinión pública y parte de la doctrina, empezó a restringir la capacidad de actuación de las Municipalities. El proceso culminó con la Sentencia dictada por el Juez DILLON seguida por la jurisprudencia posterior.

\section{La regla impuesta por el Juez John F. DILLON ${ }^{74}$ afirmaba:}

"Los poderes de las corporaciones municipales están establecidos por leyes que son de aplicación general e indiscutible. Las corporaciones locales poseen y pueden ejercitar los siguientes poderes, y no otros: Poderes expresamente conferidos por la Constitución americana, por los estatutos o la carta. Poderes necesaria o justificadamente implicitos en las facultades otorgadas. Poderes esenciales para el cumplimiento de los objetivos o propósitos declarados.»

A esto se añade:

«... ante la existencia de cualquier duda sustancial y razonable concerniente a la pertenencia o no de un poder, los tribunales deberán resolver siempre en contra de lo solicitado por la Municipality y el poder le será denegado» ${ }^{75}$.

La importancia práctica de la regla DiLlon fue que si un municipio deseaba regular una materia o una actividad debería encajarla en alguno de los poderes que antes hemos citado. La explicación que DILLON dio a su razonamiento fue la siguiente:

\footnotetext{
73 William E. Farrell, «Daley Is Silent on Seeking Sixth Term as Mayon,, New York Times, 7 de octubre de 1974, p. 22. El artículo declaraba que, pese a que sólo la mitad de la población creía en las promesas de Daley, un 84 por 100 estaban seguros de que podía llevar a cabo sus pretensiones y un 77 por 100 pensaba que estaba haciendo un buen trabajo. Nadie se atrevia a criticar su labor.

${ }^{74}$ City of Clinton v. Cedar Rapids and Missouri Railroad Company, 24 Iowa 455, 1868. Es comúnmente conocida como «Regla de Dillon». John Dillon, Juez del Tribunal Supremo de Iowa.

75 John F. Dillon, Comentarios sobre las Leyes de las Corporaciones Municipales, vol. 1, Boston, 1911, p. 448.
} 
"Ĺas corporaciones inunicipales deben su existencia y sus poderes al poder legislativo del Estado al que pertenecen. Es éste el que les da la vida y sin él no pueden existir. Asi como los crea los puede destruir. Y al igual que los puede destruir también puede reducir su poder de acción.»

Esta visón de la ciudad como elemento que se encuentra a la libre disposición del legislativo de un determinado Estado fue rechazada, como antes vimos, por otros jueces, si bien la regla DILLON se convirtió en la doctrina que se siguió y sigue aplicándose en la actualidad.

Podemos concluir diciendo que la capacidad de una Municipality para encargarse de una determinada materia depende de si las garantías para el ejercicio de ese poder emanan de los Estatutos del propio ente o de la Constitución del Estado al que pertenece y de la interpretación que los tribunales estatales hagan de dichas normas.

La estricta interpretación constitucional dada por DILLON ha sido limitada sólo en dos casos:

El primero se estableció obligando a incluir en las Constituciones de los Estados la prohibición expresa de dictar normas que afecten sólo a una ciudad. Las normas deben ser, en lo posible, comunes para todos los municipios evitando así discriminaciones y favoritismos.

Este principio se ha burlado habitualmente utilizando la técnica de la clasificación. Así, se crean estatutos que, en un principio, parecen ser de aplicación general para un conjunto de ciudades que se encuentren en una categoría predeterminada. La forma favorita de clasificación es atendiendo a la población. Muchas veces esto hace que dicha norma sólo se aplique a un centenar de personas o que sólo una ciudad esté dentro de esa clasificación tan restringida.

El segundo límite a la interpretación de la regla de DiLlon viene dado por la Constitución de los Estados Unidos. La mayoría de los casos ante los que se ha enfrentado la Corte Suprema de los EE.UU. han sido en materia electoral y que se encontraban en relación con las enmiendas 14 y 15. Uno de los casos más típicos es el conocido como Gomillion contra Lightfoot, que tuvo lugar en $1960^{76}$.

\footnotetext{
${ }^{76}$ Caso Gomillion v. Lightfoot, 364 U.S., 339, 1960. En este caso se negaba el voto a un amplio número de personas de color tras configurar los distritos electorales. Tanto el Tribunal del Distrito Federal como los otros Tribunales de Apelación aplicaron la regla DiLLon al caso. Sólo cuando éste llegó a la Corte Suprema se declaró inconstitucional tal norma.
} 
Con resoluciones como éstas se puede observar la labor importante creada por la doctrina de la Corte Suprema desde su primera resolución en la materia en 1933, en la que el Juez Cardozo explicaba: una corporación municipal, que es la creación del propio Estado al que pertenece, no encuentra en la Constitución Federal ningún privilegio o inmunidad que le pueda amparar para poder actuar en contra de la voluntad de su propio creador ${ }^{77}$.

Como resumen, podemos decir que la regla de Dillon fue y sigue siendo políticamente impracticable. La práctica deja ver una relación sutilmente equilibrada entre el Estado y sus gobiernos locales en que las ayudas locales y estatales existen paralelamente a la dirección y supervisión de los Estados en un intrincado patrón de relaciones cooperativas. Cada vez se restringe más el poder de la Cámara Legislativa de cada Estado en los casos en los que su actuación disminuye las funciones de los entes locales.

\subsubsection{El derecho de home rule 78}

La interpretación estricta de los poderes de las Municipalities dada por DILloN se ve suavizada por el derecho de autorregulación, que tanto la Constitución del Estado como los Estatutos u otras provisiones legales otorguen a la ciudad. El derecho de autorregulación (home rule) es el poder que ésta tiene de promulgar y controlar su propia Carta y de controlar lo referente a sus competencias locales.

Esto se consigue mediante la elección de un grupo de personas que se encargarán de dictar normas en las materias que sean de su competencia. En esas materias el Estado reconoce capacidad legislativa a la Municipality ${ }^{79}$.

Los argumentos en contra y a favor del derecho de home rule son numerosos. Es bueno porque libera a la Legislatura del Estado y los tribunales de las cuestiones de rutina dentro de la organización local. Las desventajas se encuentran en el temor de los ciudadanos ante un incremento en los impuestos; la dificultad para cambiar la Carta una vez adoptada, y la pérdida de uniformidad entre los diferentes municipios.

La experiencia de los más de 40 Estados que han concedido la facultad de autorregulación a alguno de sus entes locales ha demostrado que dicha

\footnotetext{
77 Williams v. Baltimor Mayor, 289 U.S., 36 a 40, 1933.

${ }^{78}$ En relación a la potestad reglamentaria en general, vid. LAVILLA RUBIRA, La participación pública en el procedimiento de elaboración de los Reglamentos en los E.U.A., Madrid, Civitas, 1991 y también vid. COMELLA DORDA, Limites del poder reglamentario en el Derecho Administrativo de los EEUU, Barcelona, Cedecs, 1997.
}

79 Vid. CLS NY Const Art IX p. 1(a). 
facultad suele ser utilizada, frecuentemente, en estas áreas: licencias y regulación de negocios y del uso de la tierra; autoorganización financiera en relación con los impuestos y en materia de créditos y préstamos solicitados; y organización de la propia estructura de gobierno para resolver mejor ciertas necesidades administrativas.

En la actualidad se ha vuelto a abrir la discusión doctrinal, esta vez en sentido inverso a la que se produjo con la revolución industrial. Se pretende mirar hacia la historia para recuperar la autonomía perdida por las ciudades como castigo a la corrupción ${ }^{80}$.

\subsubsection{Competencias de la ciudad}

Veremos qué funciones se atribuyen a las ciudades tras aplicar la Regla de Dillon y el derecho de home rule. Podemos destacar dos clases de funciones atribuidas a los entes:

Por un lado, las funciones de gobierno que el ente local ejerce como agente del Estado: seguridad, salubridad, impuestos, regulación de actividades comerciales, educación y bienestar públicos. Se incluyen aquí todos esos poderes con los que cuenta el gobierno local con independencia de que el gobierno elegido por la ciudad varíe, esto es, la ciudad siempre contará con un alcalde y unos concejales aunque las personas que ocupan estos puestos cambien. También nos referimos a la capacidad legal de la ciudad para demandar y ser demandada y a la capacidad legal de la ciudad para poder contratar y ser poseedor de bienes e inmuebles.

Por otro, nos encontramos con las funciones de propiedad que son propias de la ciudad considerada como particular por su carácter privado o corporativo: provisión de agua, producción de energía, suministro de gas y servicios de transportes. Se incluyen los poderes que los entes locales tienen sobre los servicios que prestan. Esto, en opinión de autores como MARTín, está cambiando ya que los entes locales se ven obligados a competir con el sector privado que también presta servicios a los ciudadanos ${ }^{81}$.

Si bien en algunas materias esto no es tan claro y aparecen algunas facultades que se encuentran en la llamada "zona oscura» o «tierra de na-

80 Vid. J. P. ViterutTI y G. J. Russello, «Community and American federalism: images Romantic and Real», Virginia Journal of Social Policy and the Law Association, 1997, pp. 683 a 752.

81 Vid. Nancy BuRNs, The Formation of America Local Governments, cap. I, Oxford University Press, New York, 1994. La autora valora el tema de las instituciones privadas que funcionan entre las instituciones públicas. 
die» y que en ciertos Estados se reconocen como facultades gubernamentales y en otros como funciones de propiedad.

\subsection{La organización de la ciudad: el Ayuntamiento}

Los poderes con los que cuentan las ciudades en los Estados Unidos son muy diferentes incluso dentro de cada Estado. Como dijimos, dependiendo de la trayectoria histórica seguida por la ciudad ésta contará con más o menos capacidad de autogobierno al igual que será diferente el poder conferido a la figura del alcalde. A continuación explicaremos los distintos tipos de alcaldes que pueden existir en las ciudades estadounidenses y, por ende, los tipos de ayuntamientos y las funciones de los diferentes órganos de la ciudad a tenor de los poderes depositados en la figura de ese alcalde. Podemos destacar las siguientes clases de formas de gobierno: Town Meeting (reuniones del pueblo), City Commission (comisión de la ciudad), Mayor-council (Alcalde con Consejo) y council-manager (consejo-director).

Town Meeting: es la forma utilizada en los pueblos pequeños, especialmente en Nueva Inglaterra y podemos decir que se encuentra en decadencia ${ }^{82}$. En las zonas en las que sigue utilizándose se caracteriza por ser una de las formas más puras de democracia participativa directa ${ }^{83}$.

El órgano de gobierno más importante lo componen las reuniones de todos los adultos residentes que se reúnen al menos una vez al año para decidir asuntos como los impuestos a cobrar, los materiales y bienes que se deben adquirir, el funcionamiento del servicio que prestan policías y bomberos... etc. Las reuniones se presiden por un moderador que se ha elegido previamente y las decisiones se toman a mano alzada 0 a viva voz. Si el municipio tiene mayor número de habitantes se elige a una persona que representará a la comunidad de propietarios de cada edificio en las reuniones. El resto de los ciudadanos podrá asistir con derecho a ser escuchados pero sin voto. El trabajo diario es realizado por un grupo de personas elegidas en la reunión anual (selectmen). Algunas veces se elige a una de esas personas a las que se dota de una autoridad ejecutiva algo mayor (first selectmen). Se elige también a una persona que hará las fun-

82 Vid. William G. Colman, State and Local Government and Public-Private Partnerships, Connecticut, 1989.

83 Vid. John A. FAIRLIE y Charles M. KEMEIER, Gobierno de los Condados y Administración, capítulo XX, Nueva York, 1930; John F. SLY, El gobierno en los pueblos de Massachusetts, Harvard University Press, Harvard, 1930. 
ciones de administrador que recibirá un salario adecuado a tal condición dado que los demás cargos no son remunerados.

City Commission: apareció en 1901 a consecuencia de una catástrofe natural (unas inundaciones tras un huracán) y su eficiencia hizo que continuase en esa zona y se extendiese a otros lugares ${ }^{84}$. Esta forma de Comisión se caracteriza por contar con un número escaso de miembros (de 3 a 9) que reciben el nombre de comissioners. Éstos son elegidos por toda la ciudad y pueden o no pertenecer a un partido político. Sus funciones son legislativas y ejecutivas ya que se encargan de promulgar ordenanzas y de la dirección de las medidas políticas a aplicar en la ciudad en función de las necesidades y exigencias del electorado.

Uno de los miembros de la Comisión actúa como alcalde pero sus poderes adicionales son muy limitados. Preside las reuniones de la Comisión y acude a actos públicos como representante de la ciudad. En algunos casos tiene derecho de veto y capacidad para realizar algunos nombramientos. Cada uno de los miembros que compone la Comisión preside uno de los departamentos: incendios y policía, aguas, trabajos públicos y finanzas. El número de departamentos que se crean depende siempre del número de miembros que compongan la Comisión.

Esta forma de gobierno se utiliza sobre todo en ciudades pequeñas. La concentración de poderes en cada una de las comisiones hace sencillo descubrir a los responsables de funcionamientos defectuosos. La falta de coordinación hace que se pierda la visión de la ciudad como un conjunto, como sobre todo a la hora de dictar los presupuestos que se hacen para cada comisión, lo cual produce auténticas batallas por intentar conseguir una mayor financiación. No estaría mal recordar aquí la frase acuñada por la Liga Nacional Municipal «desde el punto de vista de la financiación la forma de Comisión es como el motor de un coche que tiene acelerador pero no tiene frenos» ${ }^{85}$. Esta forma de organización entró en declive a partir de los ochenta.

\footnotetext{
84 Vid. David L. MARTtN, Running a City Hall Municipal Administration in America, Alabama, 1990, p. 67. El plan que le dio origen se conoce con el nombre de Plan Galveston por el nombre de la ciudad del Estado de Texas que lo utilizó por vez primera. En aquella época la ciudad era pequeña y sufrió unas inundaciones que provocaron una tremenda crisis ya que el alcalde no fue capaz de controlar la situación. Un acto del legislativo creó una comisión de cinco miembros (los cinco que formaban el consejo de la ciudad). Tras abolir la separación de poderes se invistió a la comisión con toda la autoridad legislativa y administrativa. Uno de los miembros de la comisión tendría la categoria de presidente-alcalde. Su posición era la de mero presidente de la comisión y no gozaba de ninguna prerrogativa adicional. El resto de los miembros de la comisión estaban a la cabeza de un departamento administrativo.
}

${ }^{85}$ Cfr. Liga Nacional MunciPal, Clases de gobierno municipal: su funcionamiento, Nueva York, 1939, p. 8. 
Mayour-council: esta forma de gobierno local es utilizada en más de la mitad de los municipios de los EE.UU. tanto en ciudades pequeñas como grandes e incluso en los pueblos. Se caracteriza por separar el poder legislativo: del que se encarga el consejo de la ciudad, del ejecutivo que recae en el Alcalde.

Los alcaldes se eligen directamente por el pueblo, y en su mayoría disfrutan del derecho de veto en la toma de decisiones.

En cuanto a los miembros del consejo, éstos pueden ser elegidos utilizando el sistema de distritos electorales o en su conjunto tomando como circunscripción electoral la ciudad.

Algunas de las actividades de la ciudad se administran mediante entidades que no están conectadas ni con el alcalde ni con el consejo. Su uso es más frecuente cuanto mayor sea la población de la ciudad. Nos referimos a las comisiones de agua, bibliotecas, plataformas para la vivienda, autoridades encargadas de los aeropuertos... Los miembros y directores de estos entes «separados» disponen de diferentes grados de independencia en cuanto a la política de actuación y la fiscal.

La diferencia más importante entre esta forma de gobierno local se suele realizar en función del poder que el alcalde ostenta en los municipios, uno se conoce como: Alcalde débil (weak mayor) y el otro como Alcalde fuerte (strong mayor). La diferencia entre uno y otro viene dada en la capacidad que el alcalde tiene a la hora de elegir al resto de los officers encargados de la dirección de los diferentes departamentos dentro del Ayuntamiento.

Council-manager: es utilizado por casi la mitad de las ciudades de los EE.UU. y es especialmente popular en las ciudades medianas (con población entre 10.000 y 250.000 habitantes) y las comunidades de clase media. Apareció a principios de siglo y es el reflejo de la mentalidad de los hombres de negocios que se encargaban de dirigir a las ciudades.

Los ciudadanos eligen a un conjunto de 5 ó 7 directores (City Council), que definirán la dirección política de la ciudad y que eligen, luego, a un conjunto de profesionales que se encargarán de las diferentes áreas de servicios que presta el ayuntamiento. Entre ellos nombran mediante votación a uno de los miembros para realizar las funciones de alcalde que serán simplemente honoríficas, y se otorgarán, generalmente, al miembro más antiguo o al que mayor número de votos haya conseguido. 
Las estructuras que anteriormente se describen son las formas «puras» de organización de las ciudades, pero cada una de ellas está sujeta a cambios en cada uno de los Estados atendiendo a las Constituciones de éstos y a los deseos de los propios municipios. Cada una de las formas de gobierno que podemos encontrar en los EE.UU. es el resultado de la tradición histórica del lugar, el pasado político, las protestas, participación y necesidades de los vecinos e incluso de los gustos personales de todos aquellos que ocuparon un cargo en el Ayuntamiento. 
